\title{
Measurements of the top quark mass using the ATLAS detector at the LHC
}

\author{
Davide Melini, on behalf of the ATLAS collaboration \\ Universidad de Granada, Spain \\ Instituto de Fisica Corpuscular (IFIC), Centro Mixto Universidad de Valencia - CSIC \\ E-mail: davide.melini@cern.ch
}

The top quark mass is a fundamental parameter of the Standard Model (SM). Thus measuring its value with the highest possible precision is important. The latest top quark mass measurements performed on data collected with the ATLAS detector are presented. In particular results on standard top mass measurements are presented for the fully hadronic and fully leptonic $t \bar{t}$ decay modes. Also top quark pole mass measurements from $t \bar{t}$ inclusive cross section and $t \bar{t}+1$ jet differential cross section are reported.

XXV International Workshop on Deep-Inelastic Scattering and Related Subjects

3-7 April 2017

University of Birmingham, $U K$ 


\section{Introduction}

The top quark is one of the elementary particles in the Standard Model (SM). It was discovered at the Tevatron by the CDF and DØ experiments [1,2]. All its properties are predicted by the SM theory, its mass being the only free parameter. It is thus important for experiments at the Large Hadron Collider (LHC) [3], such as ATLAS [4] and CMS [5], to measure it the most precisely possible.

The top quark mass is important also in consistency checks of the SM [6], as well as influencing the stability of the electro weak vacuum [7].

Quark masses are fundamental parameters of the SM Lagrangian. Since they are not free particles because of confinement, their masses are not observables. Definition of a quark mass is not unique and to be theoretically well defined, dynamical expressions are used. The most popular ones are the pole mass $\left(m_{\mathrm{top}}^{\text {pole }}\right)$ and the $\overline{\mathrm{MS}}$ scheme definitions. For the top quark mass, which is the only quark decaying before hadronisation, a kinematic mass can also be defined, the so called Monte Carlo (MC) mass. The connection between the fundamental parameter in the SM Lagrangian and the MC mass $\left(m_{\text {top }}^{\mathrm{MC}}\right)$ is not known. It has been estimated that the difference between top quark pole mass and $\mathrm{MC}$ mass is $\lesssim 1 \mathrm{GeV}$ [8].

\section{Top quark measurements in ATLAS}

The most dominant production of top quarks a the LHC is the production of top and antitop quark pairs $(t \bar{t})$. With a smaller rate, also single (anti-)top quark production happens. In the following we will focus on top quark pairs production only.

Once produced, top quarks almost always decay into a $\mathrm{W}$ boson and a $b$-quark. This last one then hadronises into a $b$-jet. W bosons can then decay into a lepton and a neutrino (leptonic decay) or into two quarks which then form two jets (hadronic decay)

For $t \bar{t}$ production, three main final state categories can be defined. They are classified on the number of leptons in the final state: semileptonic, dileptonic and fully hadronic. The fully hadronic channel has no leptons and is the one with the highest branching ratio. On the other hand, it suffers from a high background contamination and the reconstruction of the $t \bar{t}$ system from many jets is difficult. Dileptonic final state contains two leptons and is the cleanest one, since its signal can be well distinguished from multi jet background, but it has a smaller branching ratio and the presence of two neutrinos also makes the kinematic reconstruction challenging. Finally, semileptonic channel have a large branching ratio and the presence of exactly one lepton and missing transverse energy $\left(E_{\mathrm{T}}^{\text {miss }}\right)$, allows to separate its signal from the background. In this case, it is easier to reconstruct the two top quarks from their decay products.

ATLAS measured at $7 \mathrm{TeV}$ the top quark MC mass in all the $t \bar{t}$ categories $[9,10,11]$, as well as a measurement of $m_{\mathrm{top}}^{\mathrm{MC}}$ in singletop enriched category at $\sqrt{s}=8 \mathrm{TeV}[12]$. Also, a measurement of the top and anti-top quarks (MC) mass difference was performed [13]. In the following, a selection of the latest ATLAS top quark measurements is presented.

\subsection{Top quark MC mass measurements}

Typically, analysis measuring the top quark MC mass, use template methods. They make 
use of observables which are particularly sensitive to the top quark mass. Their distribution is parametrised as a function of the top quark mass, as it is defined in MC simulators. A likelihood fit is then performed and the MC mass best value is found to descrive the real data.
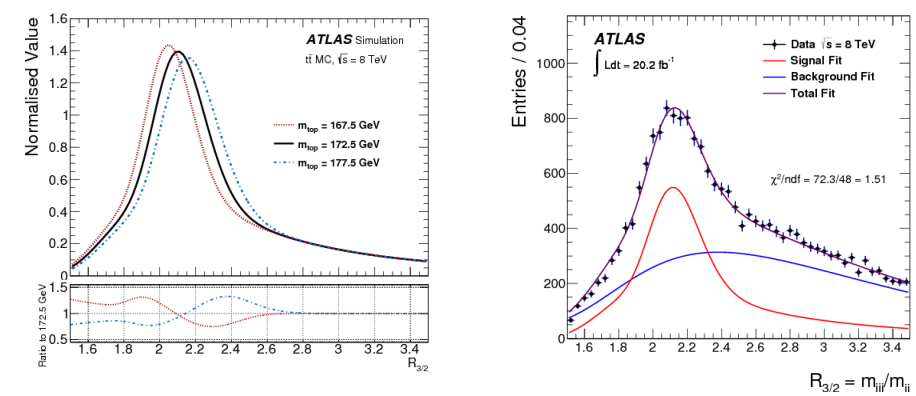

Figure 1: Dependence of $R_{3 / 2}$ on the top quark MC mass (left), and best fit to signal and background (right) [14].

In the case of the fully hadronic analysis [14], event selection requires six jets, two of which $b$-tagged, in order to reconstruct the top quark pairs. The main multi jet background is estimated using data driven techniques. The kinematic reconstruction of the system is performed thanks to a likelihood function, which simultaneously minimises the mass difference of top candidates and the mass difference between reconstructed $\mathrm{W}$ bosons and $m_{\mathrm{W}}^{\mathrm{PDG}}$. Labelling $m_{j j j}$ the mass of a top quark candidate and $m_{j j}$ the mass of a $\mathrm{W}$ boson ones, it is possible to define the observable $R_{3 / 2}=\frac{m_{j j j}}{m j j}$. It is sensitive to the top quark mass $\left(\propto m_{j j j}\right)$, as it showed in Fig. 1, while reducing the impact of jet energy scale (JES) uncertainties on the final result by using a ratio of two quantities, separately sensitive to JES.

The result obtained in this analysis is :

$$
m_{\text {top }}^{\mathrm{MC}}(\text { fully hadronic })=173.72 \pm 0.55 \text { (stat.) } \pm 1.01 \text { (syst.) } \mathrm{GeV}
$$

where the main systematic uncertainties come from JES $(0.60 \mathrm{GeV})$ and the modelling of the hadronisation of the $\mathrm{MC}(0.64 \mathrm{GeV})$.

In the dileptonic analysis [15] the invariant mass of the top quarks cannot be reconstructed, because of the presence of two neutrinos which escape detection. Thus an observable has to be chosen which does not rely on four momenta of neutrinos, such as the invariant mass of the lepton and $b$-jet system $\left(m_{\mathrm{lb}}^{\text {reco }}\right)$. Its dependence on $m_{\mathrm{top}}^{\mathrm{MC}}$ is shown in Fig. 2.

Event selection requires two oppositely charged leptons, together with at least two $b$-tagged jets. A cut is applied on the transverse momenta of the $l+b$ system to minimise the JES uncertainty and $m_{\mathrm{lb}}^{\text {reco }}$ is considered for the fit in the $[30 \mathrm{GeV}, 170 \mathrm{GeV}]$ window, to reduce the contamination from low mass resonances and increase purity and truth-matching efficiency.

For this analysis ATLAS obtains:

$$
m_{\text {top }}^{\mathrm{MC}}(\text { dileptonic })=172.99 \pm 0.41 \text { (stat.) } \pm 0.74 \text { (syst.) } \mathrm{GeV}
$$

where the main systematic uncertainties come from JES $(0.54 \mathrm{GeV})$ and $b$-jets JES $(0.30 \mathrm{GeV})$, the hadronisation modelling of the $\mathrm{MC}(0.22 \mathrm{GeV})$ and the modelling of initial and final state radiation 

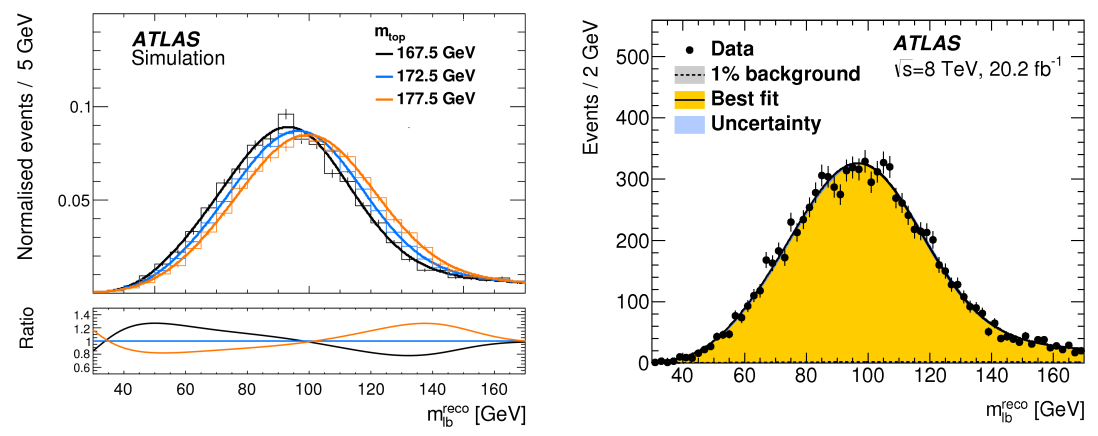

Figure 2: Dependence of $m_{\mathrm{lb}}^{\text {reco }}$ on the top quark MC mass (left), and best fit to signal (right) [15].

$(0.22 \mathrm{GeV})$. The result of this analysis has been combined with the semileptonic and dileptonic analysis at $\sqrt{s}=7 \mathrm{TeV}[9]$, resulting in a reduction of $\approx 15 \%$ on the total error.

It is in general important to have different independent analyses to measure the top quark mass, in order to benefit of their combination, reducing the total error on the extracted value. For the Run 2 of the LHC, ATLAS is going to focus more on this aspect, with a number of alternative method to extract $m_{\mathrm{top}}^{\mathrm{MC}}$. Together with the improvement of already established analysis, the aim of the ATLAS collaboration is to reach the $\approx 0.5 \mathrm{GeV}$ error on $m_{\text {top }}^{\mathrm{MC}}$.

\subsection{Top quark pole mass measurements}

With $m_{\mathrm{top}}^{\mathrm{MC}}$ measurements reaching and passing the $\approx 1 \mathrm{GeV}$ precision, it has to be noticed that $m_{\mathrm{top}}^{\mathrm{MC}}$ is not the fundamental parameter entering the SM Lagrangian. Thus, direct measurements of the top quark pole mass ( $\left.m_{\mathrm{top}}^{\text {pole }}\right)$ are also needed. The typical strategy to extract $m_{\mathrm{top}}^{\text {pole }}$ is to find observables which are sensitive and can be computed at least at NLO in perturbation theory, so that the renormalisation scheme is fixed. Such observables are then typically cross sections, either inclusive or differential.

The first observable which is used to measure $m_{\mathrm{top}}^{\text {pole }}$ is the $t \bar{t}$ inclusive cross section, $\sigma_{t \bar{t}}$. ATLAS performed such a measurement [16] for the 7 and $8 \mathrm{TeV} p p$ collisions, in the different-sign $e \mu$ final state. Comparing the measured value of $\sigma_{t \bar{t}}$ with the NNLO+NNLL theoretical prediction available $[17,18]$, as it is shown in Fig. 3, one gets:

$$
m_{\text {top }}^{\text {pole }}(t \bar{t} \text { cross section })=172.9_{-2.6}^{+2.5} \mathrm{GeV}
$$

the main source of uncertainty being coming from PDF choice $(1.8 \mathrm{GeV})$. The big uncertainty, compared to other $m_{\text {top }}^{\mathrm{MC}}$ measurements, is due to the smaller sensitivity of $\sigma_{t \bar{t}}$ on the pole mass.

Another analysis [19] has been performed with the ATLAS detector, which studies the dependence of $t \bar{t}+1$ jet differential cross section on $m_{\mathrm{top}}^{\text {pole }}$. By measuring a normalised differential cross section defined as

$$
\mathscr{R}\left(m_{\mathrm{top}}^{\text {pole }}, \rho_{s}\right)=\frac{1}{\sigma_{t \bar{t}+1} \text { jet }} \frac{\mathrm{d} \sigma_{t \bar{t}+1 \text { jet }}}{\mathrm{d} \rho_{s}} \quad \text {, with } \rho_{s}=\frac{340 \mathrm{GeV}}{\sqrt{s_{t \bar{t}+1} \text { jet }}}
$$

the sensitivity to $m_{\mathrm{top}}^{\text {pole }}$ increased, while many systematics get reduced. The observable, as it is used in the analysis, is shown in Fig. 3. Top quark pole mass extraction was possible thanks to 

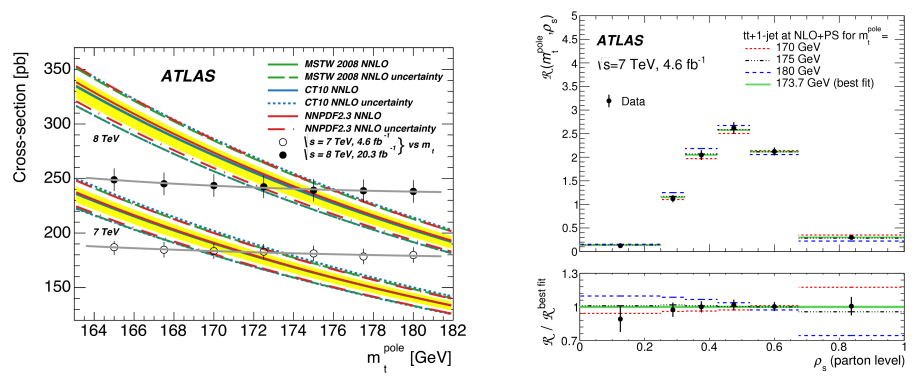

Figure 3: $m_{\mathrm{top}}^{\text {pole }}$ extracted from $\sigma_{t \bar{t}}$ (left) [16] and from $t \bar{t}+1$ jet differential distribution (right) [19].

theoretical calculation available at NLO [20]. The result of the measurement, using $7 \mathrm{TeV} p p$ collisions data only with semileptonic final state, gave:

$$
\left.\left.m_{\mathrm{top}}^{\text {pole }}(t \bar{t}+1 \text { jet diff. cross section })=173.7 \pm 1.5 \text { (stat. }\right) \pm 1.4 \text { (syst. }\right)_{-0.5}^{+0.9} \text { (theo.) } \mathrm{GeV}
$$

which have a total uncertainty very similar to the result of 2.3, but with one fourth of its luminosity. The main uncertainties on this last measurement come from JES and $b$ JES $(0.94 \mathrm{GeV})$ and ISR/FSR modelling $(0.72 \mathrm{GeV})$, but the biggest component is by far the statistical one, which can be strongly reduced using $8 \mathrm{TeV}$ data. Furthermore, the same observable can be used to measure the top quark mass in the $\overline{\mathrm{MS}}$ scheme [21].

ATLAS prospects for Run 2 are to reach the $\approx 1 \mathrm{GeV}$ total error on $m_{\mathrm{top}}^{\text {pole }}$.

\section{Summary}

The latest measurements of the top quark mass from data collected with the ATLAS detector have been reported. A summary table of all these measurementes is shown in Fig. 4. The latest ATLAS combination [22] gave a top quark MC mass of:

$$
m_{\mathrm{top}}^{\mathrm{MC}}=172.84 \pm 0.70 \mathrm{GeV}
$$

while top quark pole mass measurements reached a $2-2.5 \mathrm{GeV}$ precision.

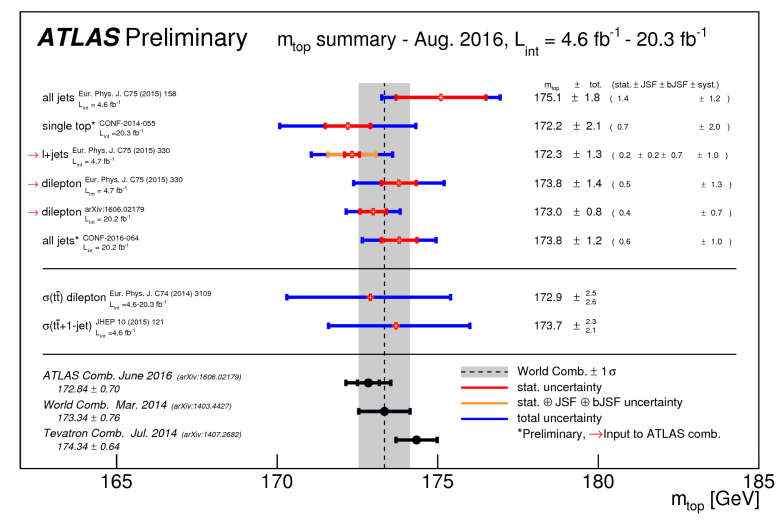

Figure 4: Summary table of ATLAS top quark mass measurements [22]. 


\section{References}

[1] CDF Collaboration, Phys. Rev. Lett. 74 (1995) 2626 [hep-ex/9503002].

[2] D0 Collaboration, Phys. Rev. Lett. 74 (1995) 2632 [hep-ex/9503003].

[3] L. Evans and P. Bryant, JINST 3 (2008) S08001.

[4] ATLAS Collaboration, JINST 3 (2008) S08003.

[5] CMS Collaboration, JINST 3 (2008) S08004.

[6] M. Baak and R. Kogler, arXiv:1306.0571 [hep-ph].

[7] S. Alekhin, A. Djouadi and S. Moch, Phys. Lett. B 716 (2012) 214 [arXiv:1207.0980 [hep-ph]].

[8] A. H. Hoang and I. W. Stewart, Nucl. Phys. Proc. Suppl. 185 (2008) 220 [arXiv:0808.0222 [hep-ph]].

[9] ATLAS Collaboration, Eur. Phys. J. C 75 (2015) , 330 [arXiv:1503.05427 [hep-ex]].

[10] ATLAS Collaboration, Eur. Phys. J. C 72 (2012) 2046 [arXiv:1203.5755 [hep-ex]].

[11] ATLAS Collaboration, Eur. Phys. J. C 75 (2015) , 158 [arXiv:1409.0832 [hep-ex]].

[12] ATLAS Collaboration, ATLAS-CONF-2014-055

[13] ATLAS Collaboration, Phys. Lett. B 728 (2014) 363 [arXiv:1310.6527 [hep-ex]].

[14] ATLAS Collaboration, arXiv:1702.07546 [hep-ex].

[15] ATLAS Collaboration, Phys. Lett. B 761 (2016) 350 [arXiv:1606.02179 [hep-ex]].

[16] ATLAS Collaboration, Eur. Phys. J. C 74 (2014), 3109 Addendum: [Eur. Phys. J. C 76 (2016) , 642] [arXiv:1406.5375 [hep-ex]].

[17] M. Czakon and A. Mitov, Comput. Phys. Commun. 185 (2014) 2930 [arXiv:1112.5675 [hep-ph]].

[18] M. Czakon, P. Fiedler and A. Mitov, Phys. Rev. Lett. 110 (2013) 252004 [arXiv:1303.6254 [hep-ph]].

[19] ATLAS Collaboration, JHEP 1510 (2015) 121 [arXiv:1507.01769 [hep-ex]].

[20] S. Alioli, P. Fernandez, J. Fuster, A. Irles, S. Moch, P. Uwer and M. Vos, J. Phys. Conf. Ser. 452 (2013), 012050.

[21] J. Fuster, A. Irles, D. Melini, P. Uwer and M. Vos, arXiv:1704.00540 [hep-ph].

[22] ATLAS Collaboration, https://twiki.cern.ch/twiki/bin/view/AtlasPublic/TopPublicResults 